\title{
Researching Scientific Structures via Joint Authorships-The Case of Virtual 3D Modelling in the Humanities
}

\author{
Sander Münster (D)
}

\begin{abstract}
One of the topics addressed by e-science research is the measurement of academic knowledge production based on electronic data and its relevance in defining the academic landscape. The author employs e-science methods to research cooperative authorships and scientific structures in a specific area of applied e-sciences: virtual 3D modelling in the humanities. Based on the findings, possibilities for crossdisciplinary and international cooperation are discussed. The number of international publications and average number of authors involved in each publication are lower than those found in other scientific fields. Moreover, research indicates that in the humanities, 3D modelling is relatively new and still emergent. Besides such general indications, several key players as people and institutions which interconnect groups of researchers could be identified on a structural level.
\end{abstract}

Keywords Cooperative authorships $\cdot 3 \mathrm{D}$ modelling $\cdot$ Humanities

\section{Introduction 1}

A major issue related to the measurement of academic knowledge production is the distinction between disciplines and the mapping of scientific structures. The vast and heterogeneous variety of possible indicators result in a lack of standardisation and homogenisation. Joint standards to measure academic performance-as intended by the German Research Council (Wissenschaftsrat: Empfehlungen zu einem Kerndatensatz Forschung Berlin 2013)_ are still being established. Our field of research is a specific area of applied e-sciences: virtual 3D modelling in the humanities. The research started with selecting a sample of publications in order to investigate current trends, scenarios and workflows in this field, and to quantify the scholarly field. The

\footnotetext{
${ }^{1}$ This article reflects the state of my research in 2015. a more recent state was presented in: Münster, S.: Digital cultural heritage as scholarly field-topics, researchers and perspectives from a bibliometric point of view. J. Comput. Cult. Heritage 12, 22-49 (2019).
}

\footnotetext{
S. Münster (殴)

Friedrich-Schiller-Universität, Jena, Germany

e-mail: sander.muenster@uni-jena.de

(C) The Author(s) 2021

C. Koschtial et al. (eds.), e-Science, Progress in IS, https://doi.org/10.1007/978-3-030-66262-2_10
} 
initial challenges were to (a) develop a suitable research instrument and to (b) perform an investigation. Due to the limitations of the included information and the magnitude of the data sample, many potentially interesting research approaches-such as a quantification of current topics, standard references and citation networks - are not applicable. The author examines the scientific community involved in this specific area and their level of cross-disciplinary and international cooperation. Furthermore, we identify the key people and institutions, which interconnect groups of researchers.

\subsection{Defining Disciplines}

To start, a definition: disciplines are characterised by common methods and theories and have similar "reference systems, disciplinary ways of thinking, quality criteria, publication habits and bodies" (Schophaus et al. 2003) as well as similar institutionalisation. Likewise, Knorr-Cetina thought that each discipline has its own "epistemic culture" in the sense of different "architectures of empirical approaches, specific constructions of the referent, particular ontologies of instruments, and different social machines" (Knorr-Cetina 1999). Although disciplines and their boundaries are results of social construction processes (Weingart 1987), a number of phenotypic fields can be identified (Knorr-Cetina 2002). One basic classification scheme is the distinction between humanities and sciences. In a more elaborate classification, the OECD distinguishes between six scientific fields containing about 40 disciplines (OECD 2002, 2007). Furthermore, especially library classification delivers highly sophisticated distinction categorisation schemes (Semenova and Stricker 2007).

\subsection{Defining Cross-Disciplinarity}

Cross-disciplinarity refers to a "confrontation of several disciplines with a [joint] topic or issue" (Schophaus et al. 2003). In regard to this, Schelsky speaks of a "partial scientific development unit at the empirical object" (Schelsky 1966). Crossdisciplinary collaboration is characterised by developing a multidisciplinary terminology and a joint methodology (Gibbons 1994; Münster et al. 2014). The degree of institutionalisation of cross-disciplinary fields ranges from temporary collaborations to the creation of new "hybrid" research disciplines (Klein 2000) such as the digital humanities, in which computing is applied to foster humanities research. 


\section{The Case of Virtual 3D Modelling in the Humanities}

\subsection{Field of Research}

3D models and visualisation have always been an important medium for teaching, illustrating and researching historical facts and items. While historical picture sources usually provide elusive and fragmentary impressions, digital three-dimensional models of historical objects and their depictions offer the chance to convey holistic and easily accessible impressions. Until 2000, virtual 3D modelling technologies and computer-generated images of cultural heritage objects were used merely as a digital substitute of physical models (Novitski 1998). Nowadays, 3D models are widely used to present historic items and structures to the public (Greengrass and Hughes 2008) as well as in research (Favro 2004) and education (El Darwich 2005). In addition, 3D technologies can obviously serve cultural heritage management and conservation tasks, and even their advertising. An important distinction needs to be drawn between still extant, no longer extant, and never realised objects. 3D modelling technologies make it possible not only to digitise historic objects which are still extant, but even to virtually reconstruct objects that are no longer extant physically and only known from descriptions. ${ }^{2}$

\section{Research design}

This investigation of scientific structures related to the usage of $3 \mathrm{D}$ modelling techniques for both extant and no longer extant types of historical objects is based on an analysis of published project reports and presentations. An upstream problem was the identification of relevant publications. Unlike, for example, in medicine, there are no comprehensive publication databases extant for cultural studies and humanities. Prior to creating the database, three experts-chairholders in the fields of archaeology, art history and geomatics - were queried to identify relevant journals and conferences. This yielded the following findings:

- On the one hand, in the field of cultural and history studies, no multidisciplinary, periodically held international conferences are known that deal specifically with $3 \mathrm{D}$ modelling. However, there are a number of local or non-periodic conferences and workshops that deal with specific questions or topics.

- On the other hand, there are four major conferences on the topic in the fields of archaeology and cultural heritage: The International Workshop for 3D Virtual Reconstruction and Visualization of Complex Architectures (3DARCH); the Computer Applications and Quantitative Methods in Archaeology Conference (CAA); the International Symposium on Virtual Reality, Archaeology and Cultural Heritage (VAST); and the Visualisation in Archaeology workshop (VIA).

\footnotetext{
${ }^{2}$ Originally published in: Münster, S., Köhler, T., Hoppe, S.: 3D modeling technologies as tools for the reconstruction and visualization of historic items in humanities. A literature-based survey. In: Traviglia, A. (ed.) Across Space and Time. Papers from the 41st Conference on Computer Applications and Quantitative Methods in Archaeology, Perth, 25-28 March 2013, pp. 430-441. Amsterdam University Press, Amsterdam (2015).
} 
- The Journal for Digital Heritage represents an overarching publication organ for digital content on all humanities.

\subsection{Data Sample}

These findings formed the basis for collecting the data sample presented in Table 1. As a scope for conference proceedings, entire volumes were included and relevant journal articles were identified via keyword search. A sample of 452 journal articles and conference proceedings was included during the first stage of the analysis. The articles selected were written in English and, for practical reasons, had to be available electronically. Especially the latter selection criterion meant that no publications of the VIA conference and only single volumes of CAA and VAST could be included. In addition to these conference papers, relevant articles from the Journal for Digital Heritage and other periodicals were included using a keyword-based search.

One major obstacle to building a research database was the fact that most of the included conferences and journals were not listed in citation repositories or in publication databases such as ISI Web of Science, Scopus, or Google Scholar in 2012 when the database was compiled. This made it necessary to retrieve metadata by crawling data from each single contribution. For each article, the following information was obtained:

- names and affiliations of contributing authors

- names and addresses of affiliated institutions

- source data (conference and publication name, year, type of document)

- title of publication.

Moreover, conference contributions were classified based on their content. As pointed out in (Münster et al. 2013), one-third (37\%) of these articles deal with neither 3D modelling nor historical objects. Nearly the same number of articles report about single projects. This means that they describe workflows for rebuilding certain historic items as 3D models. Another group of contributions deals with certain aspects

Table 1 Sample $(n=452)$

\begin{tabular}{l|l|r}
\hline Publication & Volume & No. \\
\hline 3DArch Conf. & $2005-2009$ & 112 \\
\hline CAA Conf. & 2007,2009 & 130 \\
\hline VAST Conf. & $2003-2007,2010$ & 105 \\
\hline J. Digital Heritage & From 2000 & 52 \\
\hline $\begin{array}{l}\text { Various project reports and publications dealing with no longer extant } \\
\text { objects }\end{array}$ & $1999-2011$ & 79 \\
\hline
\end{tabular}

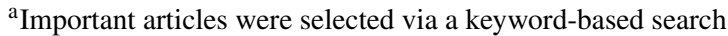


of 3D modelling for historical purposes, such as presentation and modelling strategies, data acquisition methods, or handling and classification of 3D data. Focussing on project reports only, a further investigation takes into consideration whether an original object is still extant. To quantify, more than $2 / 3$ of project reports deal with extant objects or their fragments, while another $1 / 3$ focus on non-extant objects. While digitisation of extant objects is mostly based on acquired data and uses widely automated algorithms, reconstruction of no longer extant or never realised objects usually involves manual model creation using CAD or VR software tools. For each type of object, the model creation processes are very different. For this reason, one aim here is to investigate whether both topics might attract different contributors and build slightly different sub-communities.

\subsection{Scientific Approach: Analysis of Scientific Authorship Relations}

From a disciplinary point of view, an investigation of "laws governing the production, flow and application of information in science" (Vinkler 1996) by a numerical analysis of publications is part of bibliometrics. This discipline contains a wide spectre of measures and methods to investigate scientific structures and output. Based on former categorisation and formalisation attempts (Vinkler 2001; Egghe 2009; Gauffriau et al. 2007), several bibliometric approaches are distinguishable, according to their objects of study (Table 2). Not all research approaches are applicable to the described data sample. The limiting factors are the low number of samples and the types of information collected.

The metadata of publications are a major object of study. Related approaches include classification of various attributes, like publication type, journal, disciplinary

Table 2 Brief overview of bibliometric approaches

\begin{tabular}{l|l}
\hline Object of study & Approach (example) \\
\hline Publications & $\begin{array}{l}\text { Classification (i.e. De Solla Price 1963) } \\
\text { Scaling laws (i.e. Bettencourt et al. 2008) }\end{array}$ \\
\hline Authors & $\begin{array}{l}\text { Key numbers (i.e. De Solla Price 1963) } \\
\text { Clustering of authors (i.e. Glänzel 2001) } \\
\text { Disciplinary productivity (i.e. Lotka 1926) }\end{array}$ \\
\hline Topics & $\begin{array}{l}\text { Topic graphs (i.e. Schoepflin and Glänzel 2001) } \\
\text { Scientograms (i.e. Vargas-Quesada and Moya-Anegón 2007) } \\
\text { Epidemiology of ideas (i.e. Garfield 1980) }\end{array}$ \\
\hline Citations & $\begin{array}{l}\text { Impact (i.e. Hirsch 2005; Smith 2012) } \\
\text { Co-citation analysis (i.e. Bellis 2009) }\end{array}$ \\
\hline Communities & $\begin{array}{l}\text { Structures (i.e. Newman 2001a; Glänzel and de Lange 2002) } \\
\text { Protagonists (i.e. Otte and Rousseau 2002; Newman and Girvan 2004; } \\
\text { Kretschmer and Aguillo 2004) }\end{array}$ \\
\hline
\end{tabular}


backgrounds or dates. These classes allow for comparison and evaluation of distribution functions, as well as monitoring of trends and prediction of emergent fields of research based on time rows (Bettencourt et al. 2008). While the latter approach in particular relies on plenty of lossless data, it does not seem applicable to our research.

Another important object of study is related to authors of publications. One approach is to calculate key numbers in various ways, such as an average count of authors per publication or a rate of publications authored by single individuals. As one example, the cutting-edge analyses of De Solla Price (1963) in the early 1960s employed key numbers to investigate the transformation processes of scientific production. A second approach is to cluster authors, for example by nationality, to study preferences for international joint authorships (Glänzel 2001). Both research approaches are employed in our study to investigate cooperative authorship. A third approach uses author data to measure disciplinary characteristics such as disciplinary productivity, used in this article by employing the Lotka Coefficient (Egghe 2009; Lotka 1926). Furthermore, Schubert and Glänzel studied preference patterns of cross-national authorships (Schubert and Glänzel 2006) and stated that there was a "major influence [of] historical, cultural and linguistic proximities" (p. 426). Such an approach is not applicable to this investigation due to the small number of samples.

Several investigational approaches focus on topics described in researched articles. As one example, a topic graph structure classifies current research topics in a certain scientific area (Glenisson et al. 2005; Schoepflin and Glänzel 2001). Moreover, approaches like epidemiology of ideas (Goffman and Newill 1964) or scientograms focus on predicting emergent trends based on an evolution of the importance of topics.

Over the last few years, citations have become a very popular object of research into scientific performance. This includes measuring individual impact factors via indexes, most popularly the h-index invented by Hirsch (2005), or the total impact of certain journals via the Garfield index (Vinkler 2012). Furthermore, co-citation analysis provides clues about the evolution of a scientific area over time and its standard works (Bellis 2009). Neither citations nor topics are covered by the available data, so these objects of study are not included in our investigation.

Scientific communities as a "group of scientists [...] agreed on accepting one paradigm" (Jacobs 2006) are another research object of bibliometrics. One particular approach, the study of co-authorship networks, focusses on detecting structures of scientific cooperation employing graph analysis methods (Vargas-Quesada and Moya-Anegón 2007). Although such research approaches are limited to a structure representation (Hardeman 2013) and include a number of potential sources of error and limitations, computer-based analysis and evaluation of co-authorships fosters several new insights related to scientific cooperation (De Stefano et al. 2011; Lu and Feng 2009). For example, a comprehensive investigation of publications in the fields of medicine, science and computing (Newman 2001a, b, c) reveals that the "small-world phenomenon" (Milgram 1967) (i.e. any two authors are connected in a chain of on average five to six parties) could be identified for these scientific communities. A number of smaller studies also deal with co-authorship within individual disciplines (Aleixandre-Benavent et al. 2012), or for individual countries or regions 
(Abramo et al. 2010; Morelli 1997; Gaillard 1992). Besides describing scientific networks, another issue is to identify important players as protagonists of scientific communities (Kretschmer and Aguillo 2004; Hou et al. 2007). This latter aspect is of interest regarding the community dealing with $3 \mathrm{D}$ modelling in the humanities.

\section{Findings}

\subsection{Indication 1: Cooperative Authorship}

One of the essential characteristics of modern research is the large number of authors involved in a single publication. In 1962, De Solla Price pointed out that in 1900, more than $80 \%$ of publications had a single author (De Solla Price 1963). In 2000, a study of scientific articles listed in the Science Citation Index (Glänzel et al. 2004) revealed an average contribution of 4.2 authors per article, wherein the proportion of articles written by individual authors was only $11 \%$. Within our research sample, an average of 3.4 authors was involved in each publication. From the perspective of cross-disciplinary and international cooperation, the disciplinary affiliation of the author collectives seems especially interesting. As shown in Fig. 1, the majority of the studied publications were written by authors or author collectives belonging to the same area of research and only a limited number of publications were crossdisciplinary. The author's disciplinary affiliation was identified from the correspondence addresses noted in publications. However, such data only provides information about the disciplinary focus of an employing institution and not on the author himself. To overcome this potential flaw, an alternative method which takes personal disciplinary backgrounds into account - self-sorting by authors via questionnaireis intended for the next stage of the research, but not yet realised for this set of data. In this data, for $21 \%$ of authors the respective disciplines at affiliated institutions could not be identified or distinguished precisely.

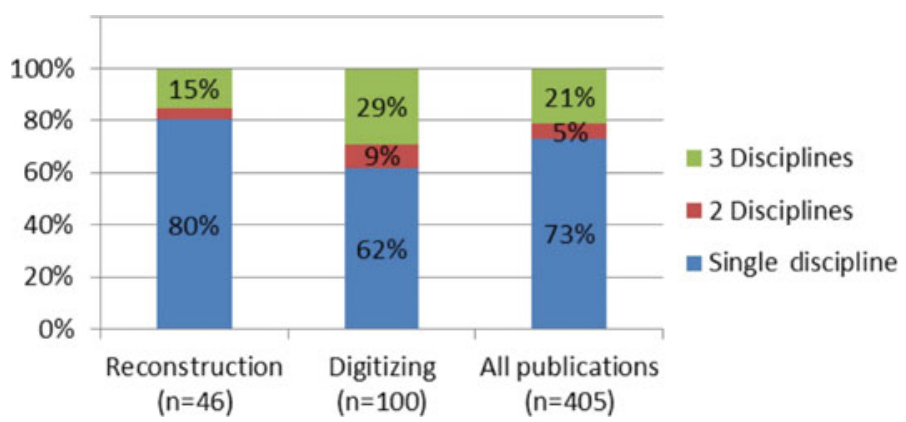

Fig. 1 Number of participating disciplines 
With regard to the distinction between types of modelling, the number of crossdisciplinary publications describing the digitisation of extant objects is significantly higher than for reconstruction projects.

Another interesting aspect is the disciplinary background of the authors' employing institutions depending on the type of object modelled. In the table shown in Fig. 2, cross-disciplinary collaborations were included proportionately and each cross-disciplinary publication has been counted with 1 , while for publications including two disciplines, each of them has been counted with 0.5 . It seems remarkable that a large number of articles describing reconstruction projects of unrealised or non-extant objects were written by authors affiliated with institutions in the field of architecture, while publications for digitisation projects were often written by authors with a background in engineering and geosciences. A plausible explanation is provided by the competence profiles of these departments. For example, automated data acquisition via remote sensing techniques is a focus of the geosciences, while architectural studies incorporate extensive know-how about both architectural history and CAD modelling. Figure 3 shows cross-disciplinary authorships in the researched publications. Each node stands for a single publication and each edge represents the disciplinary assignment of the participating authors. The graph shows that authors from institutions in the digital humanities are especially frequently involved in crossdisciplinary cooperative authorships. Preferred partners are authors from institutions in the field of computer science, while joint publication with authors from the humanities tends to occur rarely.

As shown in Fig. 4, a significant number of publications were written by authors whose employing institutions are in the same nation. Compared to findings related to other scientific domains, which estimate an overall rate of international publications at $35 \%$ (Acosta et al. 2010), the number of international publications in the sample is

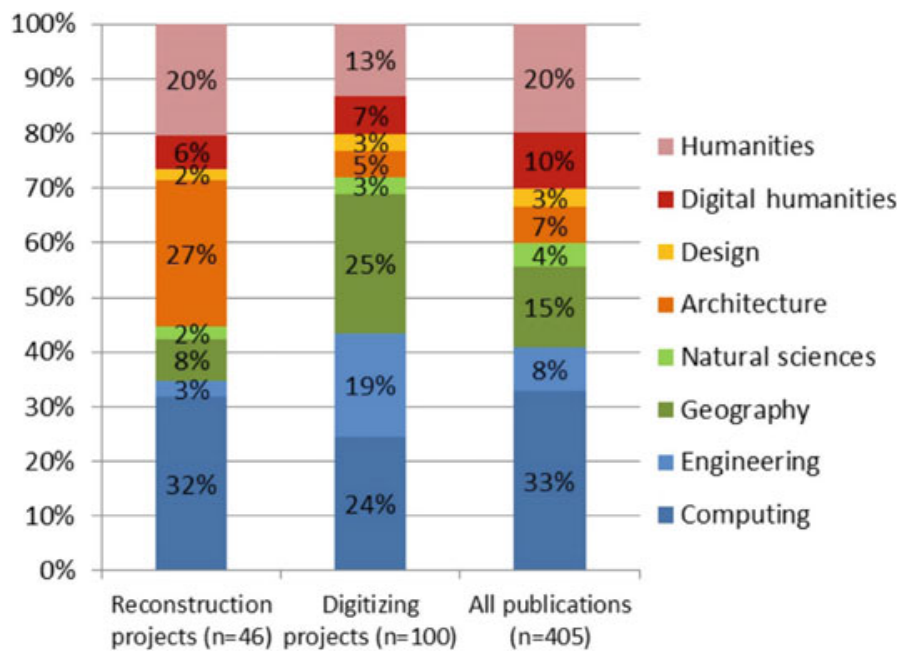

Fig. 2 Disciplinary affiliation of publication authors 


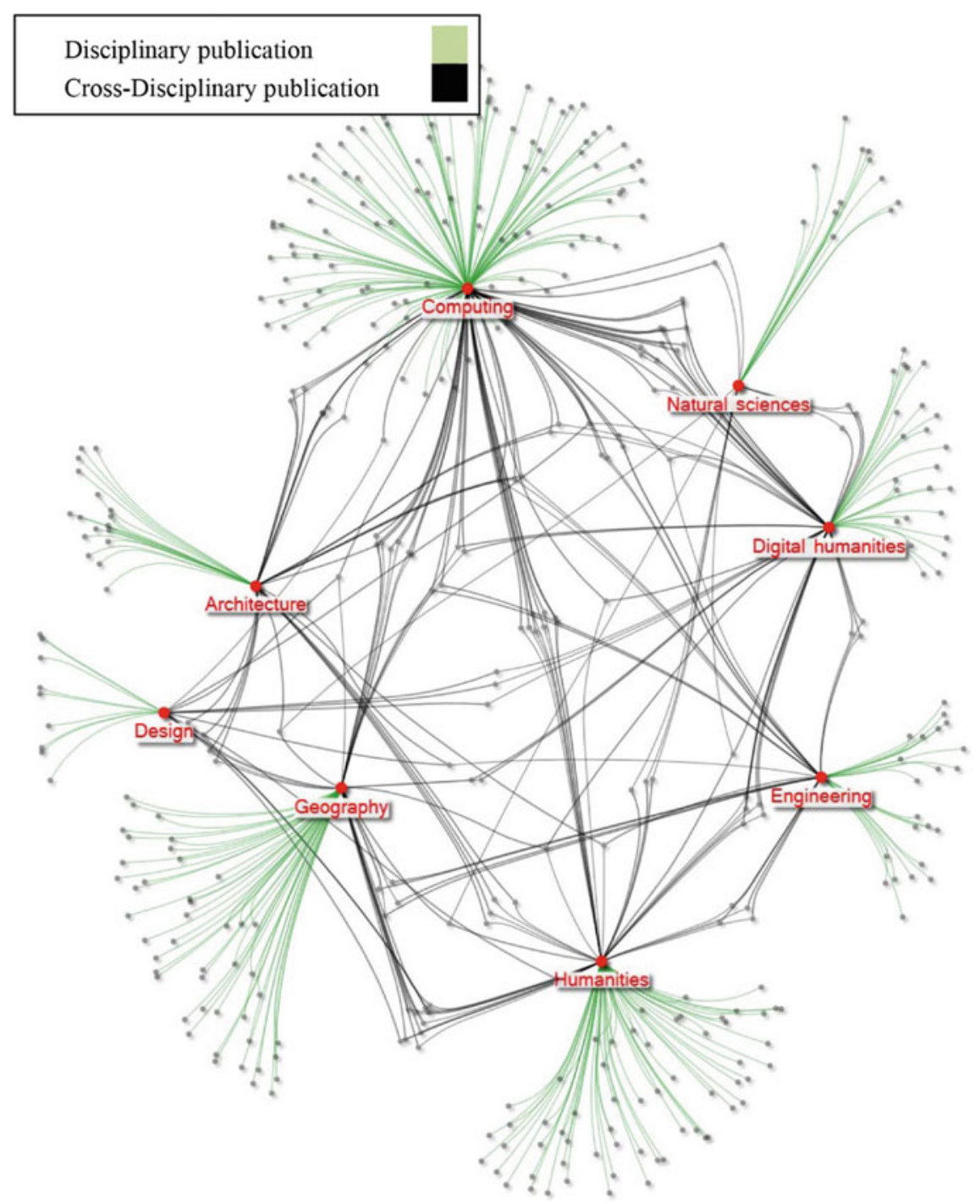

Fig. 3 Cross-disciplinary authorship

significantly lower. Analogous to the findings related to interdisciplinary cooperation, the number of international publications describing digitisation projects is above average, while only $8 \%$ of the publications describing reconstruction projects were written by international teams.

The findings of a below average rate of international and cross-disciplinary authorships in combination with a large variety of involved disciplines indicate the fuzzy 
demarcation of the field. This assumption is supported by the finding that only $30 \%$ of authors are employed in institutions which prioritise humanities or digital humanities.

\subsection{Indication 2: Lotka Coefficient}

One of the most common indicators is the number of publications per author. Relatedly, Lotka (1926) developed a distribution function for the publication frequency of individual authors which covers a wide range of disciplines and their publications. The distribution curve shows that a large number of authors with only one publication are contrasted by a very small number of authors with multiple publications.

Related to the investigated publication data, a classical Lotka distribution already revealed an extensive congruence (Fig. 5). This follows the formula

$$
Y=C / X^{n}
$$

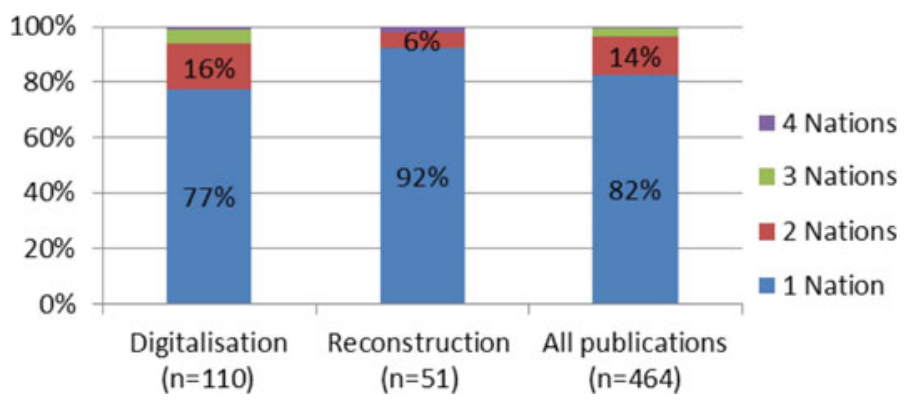

Fig. 4 Cross-national publications

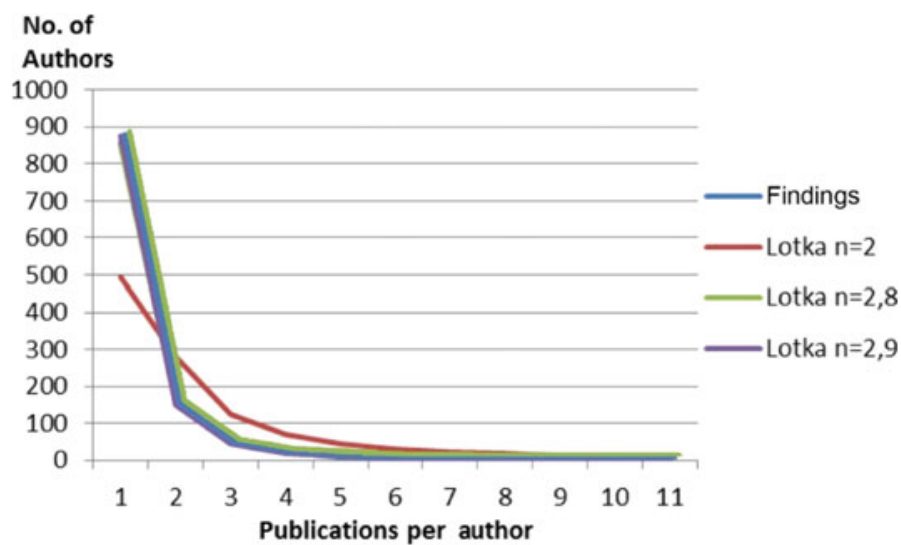

Fig. 5 Frequency distribution curves for publications 
where $\mathrm{C}$ is the total number of authors included $(n=1120)$ and $X$ indicates the number of publications of each cohort (authors with 1, 2 or more publications). The exponent $\mathrm{n}$ is a constant. From his studies, Lotka postulated an average exponent of $n=2$, which varies significantly depending on the investigated discipline (Egghe and Rousseau1990; Egghe 2000), while recent studies assumed an average value of 2.3 to 2.5 (Chung and Kolbe 1992; Pulgarín 2012). In the empirical findings, the distribution function of the investigated publications coincides with $n=2.8 \ldots 2.9$. Any further interpretation of these values must be estimated in the context of the relatively small and potentially flawed sample. Compared to the lower mean values of the exponent mostly cited in literature, the above-average exponent found here indicates low publication productivity with a disproportionate number of authors who are only occasionally involved.

\subsection{Indication 3: Key Players ${ }^{3}$}

Another hypothesis is that collaborative publications establish knowledge communication between authors. The basic idea is that, in most cases, common authorship would be related to a personal connection and interaction between all included authors. Depending on sociological role theory, such a connection between peopleregardless of its strength (Granovetter 1973) — could foster sharing and exchange of ideas and information. Regarding structure, connections between people across disciplinary and national borders play a key role in disseminating information in social communities. ${ }^{4}$ Nevertheless, information transfer in the context of joint publications is just assumed and intensity or even information transfer between authors cannot be reconstructed based on empirical data.

The sample publications were authored by 1500 individuals who were connected by over 3000 links (Fig. 6). Most of the publications were written by authors belonging to institutions of the same discipline and nationality. All the individuals at each institution were incorporated into Fig. 7. Key players were highlighted in the graphs: these were the people and institutions that were in the top ten in the categories of (a) number of connections to other authors (degree), (b) the relevance as a connecting factor between author groups (betweenness centrality) or (c) the number of publications. (Wasserman and Faust 1994) But there are also several international or cross-disciplinary networks visible whose members have written more than just one joint publication. It was possible to identify some important key players

\footnotetext{
${ }^{3}$ Originally published in: Münster, S., Köhler, T., Hoppe, S.: 3D modeling technologies as tools for the reconstruction and visualization of historic items in humanities. a literature-based survey. In: Traviglia, A. (ed.) Across Space and Time. Papers from the 41st Conference on Computer Applications and Quantitative Methods in Archaeology, Perth, 25-28 March 2013, 430-441. Amsterdam University Press, Amsterdam (2015).

${ }^{4}$ There are several studies on scientific communities and inherent social interaction, i.e. Stützer, C.: Knowledge transfer in web-based collaborative learning systems (PhD-Thesis), Dresden 2013.
} 


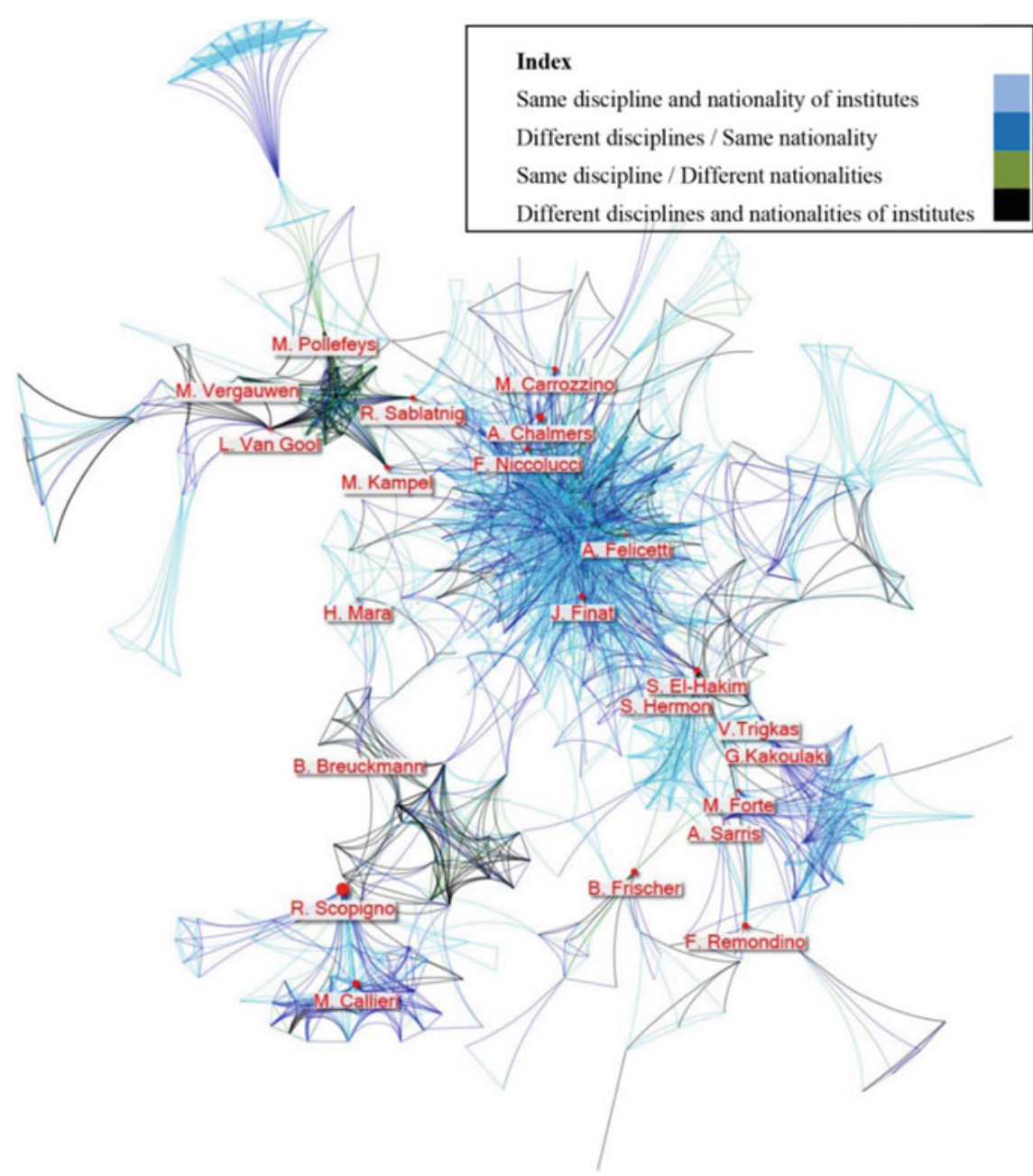

Fig. 6 Author-co-author relations—individuals (key players highlighted)

who connect groups of researchers. From an institutional perspective, the cooperation between the University of Leuven and the technical universities of Vienna and Zürich has produced a particularly large number of cross-disciplinary and international publications. A further, if smaller, cluster includes mostly French and Italian institutions, but also encompasses authors from Japan and Germany. Generally, there is a high level of networking and number of publications from people and institutions working on data-based visualisation. Finally, the key players are most connected, both internationally and cross-disciplinarily. To validate this, the results were discussed with experts. Generally, these key players are not only active publishers, but often 


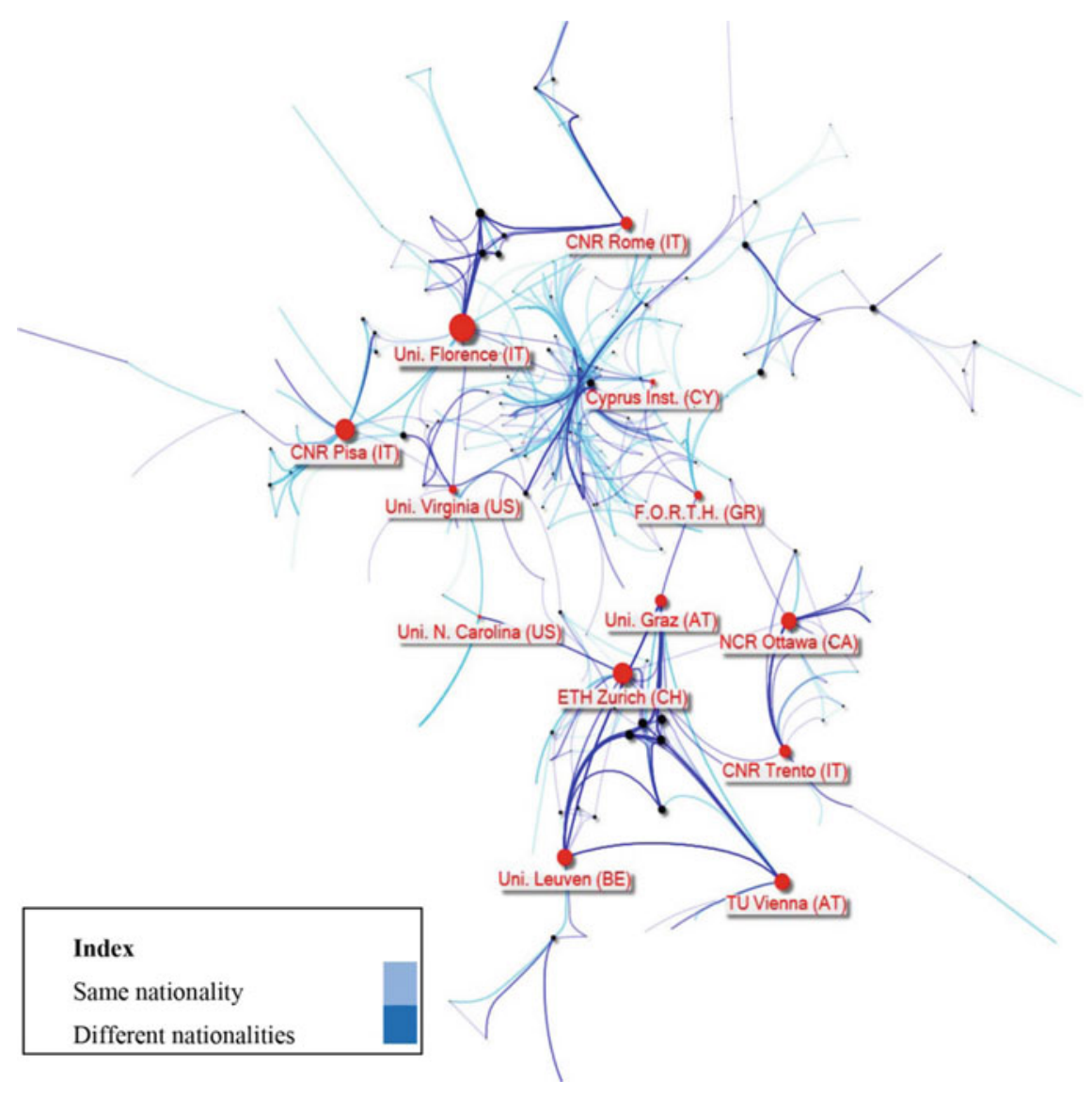

Fig. 7 Author-co-author relations-institutions (key institutions highlighted)

also play key roles in the community in other ways, too, whether as members of scientific committees, conference chairs, and initiators or leaders of projects.

We also investigated the connection between theory and practice in the field of 3D modelling in the humanities. We compared, for each institution, the number of participating digitisation and reconstruction projects described in articles and the number of publications. The results show that institutions with a high publication output are usually also involved in an exceptionally large number of projects. A significant difference between ranks of publication activity and project participation was identified in just a few institutions, such as the TU Wien or the Istituto di Scienza e Tecnologie dell'Informazione (Table 3).

This leads to the assumption that a scientific community is primarily a community of practice (Lave and Wenger 1991), with a close link between practical project work and theory, while specific think tanks as theory building institutions are currently not visible for this field of research. 
Table 3 Ranks of project and publication participation by institution

\begin{tabular}{l|l|l}
\hline Institution & Projects (Rank) & Publications (Rank) \\
\hline Politecnico di Milano & $10(1)$ & $14(1)$ \\
\hline ETH Zürich & $8(2)$ & $12(4)$ \\
\hline National Research Council Canada & $7(3)$ & $11(5)$ \\
\hline University of Florence & $7(4)$ & $12(3)$ \\
\hline Istituto di Scienza e Tecnologie dell'Informazione & $\mathbf{6 ( 5 )}$ & $\mathbf{1 3}(\mathbf{2})$ \\
\hline Centre for Scientific and Technological Research & $6(6)$ & $7(7)$ \\
\hline University of Virginia & $5(7)$ & $6(14)$ \\
\hline Tokyo Denki University & $4(8)$ & $6(12)$ \\
\hline CNR Institute of Technology Applied to Cult. Heritage & $4(9)$ & $6(15)$ \\
\hline TU Wien & $\mathbf{4 ( 1 0 )}$ & $\mathbf{1 1 ( 6 )}$ \\
\hline
\end{tabular}

\section{Conclusion}

With regard to the aim of identifying scientific structures via co-authorships, it was found that the field of 3D modelling in the humanities at an international level is widely dominated by research interests and approaches from archaeology and cultural heritage research. However, the authors involved come from a large variety of disciplinary backgrounds.

Another finding is that the number of publications written by international teams and average number of authors involved in each publication are lower than in other scientific fields. It seems remarkable that publications about digitisation projects which deal with extant objects are significantly more often written by crossdisciplinary and international teams than publications describing a reconstruction of no longer extant or never realised objects. This may be caused by the slightly different disciplinary constitutions and uses of publications in digitisation and reconstruction projects. Taking the relatively small and potentially flawed sample into account, further investigations and additional data are required for a valid evaluation.

3D modelling in the humanities is relatively new and emergent field of research. This is indicated by the above-average coefficient for a Lotka distribution describing the frequency of publications per author. Nevertheless, contributors from various disciplines were involved in the researched publications, which may indicate a currently blurry demarcation of the scientific field. Even if these findings are endorsed by other studies (Albrecht 2013), both indications provide only a hint that the field is becoming established.

What are the implications for research on e-sciences? This article described several strategies for investigating scientific structures in the field of 3D modelling in the humanities, based on electronic data and using software tools for graph analysis and QDA software for qualitative content analysis. Information was retrieved about structures and publication practices in the field. It was found that the investigated publications were mostly about archaeology and cultural heritage, while other research 
interests like aspects of cultural or art history were treated mostly via national communities and published in offline media. While the research objects and issues are closely related to the humanities, just a minority of authors are affiliated with (digital) humanities, and authors with a background in computing are very prominent in the publications. Even though these findings require further investigation, they may indicate that an international community in digital humanities is less influenced by practitioners whose competence relates to the research questions and objects than by those who provide digital research methods.

\section{References}

Abramo, G., D'Angelo, C.A., Solazzi, M.: The relationship between scientists' research performance and the degree of internationalization of their research. Scientometrics 86, 629-643 (2010)

Acosta, M., Coronado, D., Ferrándiz, E., León, M.D.: Factors affecting inter-regional academic scientific collaboration within Europe. the role of economic distance. Scientometrics 87, 63-74 (2010)

Albrecht, S.: Adoption of eScience Practices among Scholars in the Humanities and Social Sciences (Presentation). 2nd Network Conference des E-Science Forschungsnetzwerks Sachsen, June 1113, 2013, Dresden (2013)

Aleixandre-Benavent, R., Aleixandre-Tudo, J.L., Alcaide, G.G., Ferrer-Sapena, A., Aleixandre, J.L., Toit, W.d.: Bibliometric analysis of publications by South African viticulture and oenology research centres. S. Afr. J. Sci. 108, 74-84 (2012)

Bellis, N.D.: Bibliometrics and Citation Analysis from the Science Citation Index to Cybermetrics, Plymouth (2009)

Bettencourt, L.M.A., Kaiser, D.I., Kaur, J., Castillo-Chávez, C., Wojick, D.E.: Population modeling of the emergence and development of scientific fields. Scientometrics 75, 495-518 (2008)

Chung, K.H., Kolbe, P.T.: Empirical regularities in the market for real estate research output. J Real Estate Res 7, 115-124 (1992)

De Solla Price, D.: Little Science-Big Science. Columbia University Press, New York (1963)

De Stefano, D., Giordano, G., Vitale, M.: Issues in the analysis of co-authorship networks. Qual. Quant. 45, 1091-1107 (2011)

Egghe, L.: A heuristic study of the first-citation distribution. Scientometrics 48, 345-359 (2000)

Egghe, L.: Performance and its relation with productivity in Lotkaian systems. Scientometrics 81, 567-585 (2009)

Egghe, L., Rousseau, R.: Introduction to Informetrics. Quantitative Methods in Library, Documentation and Information Science. Elsevier, Amsterdam (1990)

El Darwich, R.: Modelle. In: Pandel, H.-J., Becher, U.A.J. (eds.) Handbuch Medien im Geschichtsunterricht, pp. 580-591. Wochenschau-Verlag, Schwalbach (2005)

Favro, D.: In the eyes of the beholder. Virtual Reality re-creations and academia. In: Haselberger, L., Humphrey, J., Abernathy, D. (eds.) Imaging Ancient Rome: Documentation, Visualization, Imagination: Proceedings of the 3rd Williams Symposium on Classical Architecture, Rome, 20. 23 May 2004, pp. 321-334. J. Rom. Archaeolo, Portsmouth (2006)

Gaillard, J.: Scientific production in developing countries. Scientometrics 23, 57-73 (1992)

Garfield, E.: The epidemology of knowledge and the spread of scientific information. Essays of an information scientist 4, 586-591 (1980)

Gauffriau, M., Larsen, P.O., Maye, I., Roulin-Perriard, A., Ins, M.: Publication, cooperation and productivity measures in scientific research. Scientometrics 73, 175-214 (2007) 
Gibbons, M.: The New Production of Knowledge: The Dynamics of Science and Research in Contemporary Societies. Sage, London (1994)

Glänzel, W.: National characteristics in international scientific co-authorship relations. Scientometrics 51, 69-115 (2001)

Glänzel, W., De Lange, C.: A distributional approach to multinationality measures of international scientific collaboration. Scientometrics 54, 75-89 (2002)

Glänzel, W., Schubert, A.: Analyzing scientific networks through co-authorship. In: Moed, H.F., Glänzel, W., Schmoch, U. (eds.) Handbook of Quantitative Science and Technology Research. The Use of Publication and Patent Statistics in Studies of S\&T Systems, pp. 257-276. Kluwer Academic Publishers, Dordrecht (2004)

Glenisson, P., Glänzel, W., Janssens, F., De Moor, B.: Combining full text and bibliometric information in mapping scientific disciplines. Inf. Process. Manag 41, 1548-1572 (2005)

Goffman, W., Newill, V.A.: Generalization of epidemic theory, an application to the transmission of ideas. Nature 204, 225-228 (1964)

Granovetter, M.S.: Strength of the weak ties. Am. J. Sociol. 78, 1360-1380 (1973)

Greengrass, M., Hughes, L.M.: The Virtual Representation of the Past. Ashgate, Aldershot (2008)

Hardeman, S.: Organization level research in scientometrics: A plea for an explicit pragmatic approach. Scientometrics 94, 1175-1194 (2013)

Hirsch, J.E.: An index to quantify an individual's scientific research output. Proc. Natl. Acad. Sci. U. S. Am 102, 16569-16572 (2005)

Hou, H., Kretschmer, H., Liu, Z.: The structure of scientific collaboration networks in scientometrics. Scientometrics 75, 189-202 (2007)

Jacobs, S.: Models of scientific community. Charles Sanders Peirce to Thomas Kuhn. Interdisc. Sci. Rev. 31, 163-173 (2006)

Klein, J.T.: A conceptual vocabulary of interdisciplinary science. In: Weingart, P., Stehr, N. (eds.) Practising Interdisciplinarity, pp. 3-24. University of Toronto Press, Toronto (2000)

Knorr-Cetina, K.: Epistemic Cultures. How the Sciences Make Knowledge. Harvard University Press, Cambridge (1999)

Knorr-Cetina, K.: Die Fabrikation von Erkenntnis. Frankfurt a. M, Suhrkamp (2002)

Kretschmer, H., Aguillo, I.F.: Visibility of collaboration on the web. Scientometrics 61, 405-426 (2004)

Lave, J., Wenger, E.: Situated Learning: Legitimate Peripheral Participation. Cambridge University Press, Cambridge (1991)

Lotka, A.J.: The frequency distribution of scientific productivity. J. Wash. Acad. Sci. 16, 317-323 (1926)

Lu, H., Feng, Y.: A measure of authors centrality in co-authorship networks based on the distribution of collaborative relationships. Scientometrics 81, 499-511 (2009)

Milgram, S.: The small-world problem. Psychol. Today 1, 60-67 (1967)

Morelli, W.V.: Computergeschütze Bildrecherche und Bildanalyse. In: Kohle, H. (ed.) Kunstgeschichte digital. Eine Einführung für Praktiker und Studierende. Warburg, Berlin (1997)

Münster, S.: Digital cultural heritage as scholarly field-topics, researchers and perspectives from a bibliometric point of view. J. Comput. Cult. Heritage 12, 22-49 (2019)

Münster, S., Köhler, T., Hoppe, S.: 3D modeling technologies as tools for the reconstruction and visualization of historic items in humanities. A literature-based survey. In: Traviglia, A. (ed.) Across Space and Time. Papers from the 41st Conference on Computer Applications and Quantitative Methods in Archaeology, Perth, 25-28 March 2013, pp. 430-441. Amsterdam University Press, Amsterdam (2015)

Münster, S., Schlenker, L., Köhler, T.: Common grounds and representations in cross-disciplinary processes. In: Carlucci, D., Schiuma, G., Spender, J. (eds.) Knowledge and Management Models for Sustainable Growth, pp. 579-589. Institute of Knowledge Asset Management, Basilicata (2014)

Newman, M.E.J.: The structure of scientific collaboration networks. Proc. Natl. Acad. Sci. U. S. Am. 98, 404-409 (2001a) 
Newman, M.E.J.: Scientific collaboration networks. I. Network construction and fundamental results. Phys. Rev. E 64, 016131 (2001b)

Newman, M.E.J.: Scientific collaboration networks. II. Shortest paths, weighted networks, and centrality. Phys. Rev. E 64, 016132 (2001c)

Newman, M.E., Girvan, M.: Finding and evaluating community structure in networks. Phys. Rev. E 69, (2004)

Novitski, B.J.: Rendering Real and Imagined Buildings: The Art of Computer Modeling from the Palace of Kublai Khan to Le Corbusier's Villas. Rockport Pub, Gloucester (1998)

Organisation for Economic Co-operation and Development: Frascati Manual. Proposed Standard Practice for Surveys on Research and Experimental Development. OECD Publications Service, Paris (2002)

Organisation for Economic Co-operation and Development: Revised Field of Science and Technology (FOS) Classification in the Frascati Manual, Paris (2007)

Otte, E., Rousseau, R.: Social network analysis: A powerful strategy, also for the information sciences. J. Inf. Sci. 28, 441-453 (2002)

Pulgarín, A.: Dependence of Lotka's law parameters on the scientific area. Malays. J. Libr. Inf. Sci 17, 41-50 (2012)

Schelsky, H.: Das Zentrum für interdisziplinäre Forschung. In: Mikat, P., Schelsky, H. (eds.) Grundzüge einer neuen Universität, pp. 71-87. Bertelsmann, Gütersloh (1966)

Schoepflin, U., Glänzel, W.: Two decades of scientometrics—an interdisciplinary field represented by its leading journal. Scientometrics 50, 301-312 (2001)

Schophaus, M., Dienel, H.-L., von Braun, C.-F.: Von Brücken und Einbahnstraßen. Aufgaben für das Kooperationsmanagement interdisziplinärer Forschung (Discussion paper Nr. 08/03), Berlin (2003)

Schubert, A., Glänzel, W.: Cross-national preference in co-authorship. Scientometrics 69, 409-428 (2006)

Semenova, E., Stricker, M.: Eine Ontologie der Wissenschaftsdisziplinen. Entwicklung eines Instrumentariums für die Wissenskommunikation. In: Ball, R. (ed.) Wissenschaftskommunikation der Zukunft, 4. Konferenz der Zentralbibliothek im Forschungszentrum Jülich, 6. 8. 11. 2007, vol. Band 18, pp. 61-69. Schriften des Forschungszentrums Jülich, Jülich (2007)

Smith, D.R.: Impact factors, scientometrics and the history of citation-based research. Scientometrics 92, 419-427 (2012)

Stützer, C.: Knowledge transfer in web-based collaborative learning systems (PhD-Thesis), Dresden (2013)

Vargas-Quesada, B., Moya-Anegón, F.D.: Visualizing the Structure of Science. Springer, Berlin [u. a.] (2007)

Vinkler, P.: Some practical aspects of the standardization of scientometric indicators. Scientometrics 35, 237-245 (1996)

Vinkler, P.: An attempt of surveying and classifying bibliometric indicators for scientometric purposes. Scientometrics 50, 539-544 (2001)

Vinkler, P.: The Garfield impact factor, one of the fundamental indicators in scientometrics. Scientometrics 92, 471-483 (2012)

Wasserman, S., Faust, K.: Social Network Analysis. Cambridge University Press, Cambridge (1994)

Weingart, P.: Interdisziplinarität als List der Institutionen. In: Kocka, J. (ed.) Interdisziplinarität.

Praxis - Herausforderung - Ideologie, pp. 159-166. Suhrkamp, Frankfurt a. M. (1987)

Wissenschaftsrat: Empfehlungen zu einem Kerndatensatz Forschung Berlin (2013) 
Open Access This chapter is licensed under the terms of the Creative Commons Attribution 4.0 International License (http://creativecommons.org/licenses/by/4.0/), which permits use, sharing, adaptation, distribution and reproduction in any medium or format, as long as you give appropriate credit to the original author(s) and the source, provide a link to the Creative Commons license and indicate if changes were made.

The images or other third party material in this chapter are included in the chapter's Creative Commons license, unless indicated otherwise in a credit line to the material. If material is not included in the chapter's Creative Commons license and your intended use is not permitted by statutory regulation or exceeds the permitted use, you will need to obtain permission directly from the copyright holder.

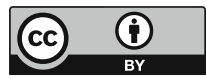

